\title{
Risk of Cesarean Delivery for Women with Obesity Using a Standardized Labor Induction Protocol
}

\author{
Rebecca F. Hamm, MD, MSCE ${ }^{1,2}$ Christina P. Teefey, MD ${ }^{3}$ Cara D. Dolin, MD, MPH ${ }^{1}$ \\ Celeste P. Durnwald, MD ${ }^{1}$ Sindhu K. Srinivas, MD, MSCE ${ }^{1,2}$ Lisa D. Levine, MD, MSCE ${ }^{1}$
}

\footnotetext{
${ }^{1}$ Department of Obstetrics and Gynecology, University of Pennsylvania Perelman School of Medicine, Philadelphia, Pennsylvania

2 Leonard Davis Institute of Health Economics, Perelman School of Medicine, Philadelphia, Pennsylvania

${ }^{3}$ Center for Fetal Diagnosis and Treatment, Children's Hospital of Philadelphia, Philadelphia, Pennsylvania
}

Am J Perinatol 2021;38:1453-1458.
Address for correspondence Rebecca Feldman Hamm, MD, Department of Obstetrics and Gynecology, Hospital of the University of Pennsylvania, 3400 Spruce Street, 2 Silverstein, Philadelphia, PA 19104 (e-mail: Rebecca.feldmanhamm@uphs.upenn.edu).

\begin{abstract}
Keywords

- obesity

- induction of labor

- cesarean delivery

- failed induction

- labor protocol
\end{abstract}

Objective We aimed to determine the risk of cesarean among women with obesity undergoing labor induction within a prospective trial that utilized a standardized labor protocol.

Study Design This was a secondary analysis of a randomized trial of induction methods. Term ( $\geq 37$ weeks) women with intact membranes undergoing induction with an unfavorable cervix (Bishop's score $\leq 6$ and dilation $\leq 2 \mathrm{~cm}$ ) were included. The trial utilized a labor protocol that standardized induction and active labor management, with recommendations for interventions at particular time points. Only women with a recorded body mass index (BMI) at prenatal care start were included in this analysis. The primary outcome was cesarean delivery compared between obese $\left(\geq 30 \mathrm{~kg} / \mathrm{m}^{2}\right)$ and nonobese $\left(<30 \mathrm{~kg} / \mathrm{m}^{2}\right)$ women. Indication for cesarean was also evaluated.

Results A total of 465 women were included: 207 (44.5\%) obese and 258 (55.5\%) nonobese. Women with obesity had a higher risk of cesarean compared with women without obesity ( 33.3 vs. $23.3 \%, p=0.02$ ), even when adjusting for parity, weight change over pregnancy, and indication for induction (adjusted relative risk [aRR] $=1.79,95 \%$ confidence interval [Cl]: [1.34-2.39]). Compared with women without obesity, women with obesity had a higher risk of failed induction (47.8 vs. $26.7 \%$, $p=0.01)$ without a difference in arrest of active phase $(p=0.39)$, arrest of descent $(p=0.95)$ or fetal indication $(p=0.32)$, despite adherence to a standardized labor protocol.

Conclusion Compared with women without obesity, women with obesity undergoing an induction are at increased risk of cesarean, in particular a failed induction, even within the context of standardized induction management. As standardized practices limit provider variation in labor management, this study may support physiologic differences in labor processes secondary to obesity. received

October 8, 2020

accepted after revision

June 17, 2021

published online

July 19, 2021 (c) 2021. Thieme. All rights reserved.

Thieme Medical Publishers, Inc., 333 Seventh Avenue, 18th Floor, New York, NY 10001, USA
DOI https://doi.org/

10.1055/s-0041-1732459.

ISSN 0735-1631. 


\section{Key Points}

- Even with a standardized induction protocol, women with obesity are at higher risk of cesarean.

- In particular, women with obesity are at increased risk of cesarean for failed induction.

- These findings support a possible biologic relationship between obesity and failed induction.

Obesity is a critical health issue in the reproductive population. In addition to antenatal complications, such as spontaneous abortion, recurrent miscarriage, gestational diabetes, preeclampsia, cardiac dysfunction, and stillbirth, multiple retrospective analyses have demonstrated that women with obesity are more likely to undergo cesarean delivery. ${ }^{1-6}$ This finding is most pronounced among women with obesity undergoing labor induction. In a systematic review evaluating women undergoing labor induction, cesarean delivery was $80 \%$ more likely among women with obesity compared with women of normal weight. ${ }^{7}$ This and other work have consistently demonstrated longer length of induction, higher prostaglandin, and oxytocin doses, as well as higher rates of cesareans for failed induction and arrest disorders. ${ }^{7-9}$

The basis for differences in labor induction outcomes among women with obesity is not entirely clear. Possible explanations include (1) physiologic differences and (2) care variation. One method to reduce care variation in medicine is to structure clear practice guidelines. In theory, such standardization of care would minimize differences in clinician management that contribute to disparate outcomes. There are minimal data surrounding the impact of variation in labor induction practices by body mass index (BMI) on mode of delivery and obstetric morbidity.

In this work, we aimed to compare cesarean delivery rates among women with and without obesity in a controlled environment, in the context of a randomized trial that utilized a strict labor induction protocol. We hypothesized that differences in cesarean delivery rate by BMI would remain despite minimizing variation in labor management, adding support to a physiologic relationship between obesity and failed labor induction.

\section{Materials and Methods}

We performed a secondary analysis of a randomized clinical trial that compared time to delivery among four different induction methods (misoprostol alone, the cervical Foley alone, misoprostol/the cervical Foley concurrently, and the cervical Foley/oxytocin concurrently). ${ }^{10}$ This study was performed at the Hospital of the University of Pennsylvania from May 2013 to June 2015. This study was approved by the institutional review board at the University of Pennsylvania and all women provided written consent before participation in this study.

Women were included in the original randomized trial if they had labor induced for any indication and met the following inclusion criteria: full term ( $\geq 37$ weeks), $\geq 18$ years of age in cephalic presentation, with intact amniotic membranes, and an unfavorable cervical examination (Bishop's score $\leq 6$ and cervical dilation $\leq 2 \mathrm{~cm}$ ). Women were exclud- ed from the study if they had a prior cesarean delivery, major fetal anomaly, or had HIV, HELLP (hemolysis elevated liver enzymes low platelets) syndrome, eclampsia, or severe fetal growth restriction with abnormal umbilical artery dopplers. ${ }^{10}$ For the purpose of this secondary analysis, we also excluded women who did not have a BMI at the start of prenatal care recorded.

Women enrolled in the original trial had their induction and labor managed with a standardized protocol specific to the cervical ripening method. The protocol was derived by two of the investigators (L.D.L. and S.K.S.) and approved by an institutional obstetrical committee prior to initiation of the randomized trial. It was modeled after prior studies that utilized an active management protocol. ${ }^{11-15}$ The protocol was used for induction management, as well as latent (defined as the stage of labor prior to 6 - $\mathrm{cm}$ dilation) and active labor (defined as the stage of labor at $\geq 6 \mathrm{~cm}$ ). The cervical ripening method was the only variable that differed among women. The protocol had multiple components include the following:

- Regular cervical examinations to assess for labor progress: every 3 hours in latent labor if cervical ripening agent being used remains in place, every 2 to 4 hours in latent labor if oxytocin was being utilized, and every 1 to 2 hours in active labor.

- Interventions if labor was not progressing which included, for example, initiation of oxytocin, amniotomy, and use of intrauterine pressure catheter.

- If the woman had not yet had membranes ruptured and cervix was $\geq 4$-cm dilated, it was recommended that an amniotomy be performed at this time, if clinically feasible.

- Once cervical dilation was $\geq 5 \mathrm{~cm}$, the four individual cervical ripening protocols converged and providers were instructed to proceed with the "active labor protocol," although active labor itself was defined as cervical dilation $\geq 6 \mathrm{~cm}$ for the purposes of defining arrest disorders. The cervical ripening method was the only thing that could differ among women and each protocol was tailored for the specific cervical ripening method chosen. ${ }^{10}$ Lastly, within the labor protocol group, determination of the need for a cesarean for failed induction or arrest disorders was based off of the 2012 NICHD (National Institute of Child Health and Human Development) guidelines. ${ }^{16}$ Failed induction was defined as "failure to generate regular (e.g., every 3 minutes) contractions and cervical change after at least 24 hours of oxytocin administration, with artificial membrane rupture if feasible."16

For the purposes of this analysis, women were grouped by BMI. The Institute of Medicine (IOM) definitions were used for obesity: women with obesity $\left(\geq 30 \mathrm{~kg} / \mathrm{m}^{2}\right)$ versus 
without obesity $\left(<30 \mathrm{~kg} / \mathrm{m}^{2}\right)$. BMI was determined by direct measurement at initial prenatal visit, regardless of gestational age. The primary outcome was cesarean delivery for any indication. Secondary outcomes included indication for cesarean, time to cesarean, total length of labor, and composite maternal morbidity (defined as $\geq 1$ of the following during labor, delivery, or in the 4 weeks postpartum: third/fourth-degree perineal laceration, blood transfusion, endometritis, wound separation-infection [defined by the need for additional wound closure or the need for antibiotics], venous thromboembolism, hysterectomy, intensive care unit admission, or death). Time to cesarean was calculated as time from placement of first induction agent to time at cesarean start in hours. Total length of labor was calculated as time from placement of first induction agent to delivery time.

Bivariate comparisons of demographic and baseline clinical characteristics, as well as labor and delivery outcomes, were performed with Fisher's exact tests and Chi-square tests for categorical variables, and $t$-tests or Wilcoxon's ranksum tests for continuous variables, where appropriate. Poisson's regression with robust variance was utilized to adjust for confounders; results are presented as relative risks (RRs). We limited covariates in the model to those demonstrated to be statistical confounders: demographic and clinical characteristics that were associated on bivariate tests $(p<0.10)$ with both the group/exposure variable (BMI category) and the outcome variable (cesarean delivery). Thus, parity, race, chronic hypertension, weight change over pregnancy, indication for induction, and birth weight were assessed as possible confounders. Study group, although not associated with BMI category, was evaluated but found not to be significant in multivariable modeling. Backward stepwise elimination of covariates (with $p>0.10$ for removal) was performed to determine which covariates would be retained in the final model. Statistical analyses were performed with Stata 15 (StataCorp, College Station, TX). This study was performed with a fixed sample size determined by those who enrolled in the original trial. Based on the fixed sample size of 491 and a baseline cesarean rate of $25 \%$, we had more than $80 \%$ power to detect a 1.5 -fold difference in cesarean delivery.

\section{Results}

Of the 491 women included in the original randomized controlled trial, 465 had a recorded BMI at the start of prenatal care and were included in this analysis. Of those, $207(44.5 \%)$ were classified as obese (BMI $\left.\geq 30 \mathrm{~kg} / \mathrm{m}^{2}\right)$ and 258 (55.5\%) were classified as nonobese $\left(\mathrm{BMI}<30 \mathrm{~kg} / \mathrm{m}^{2}\right)$.

Demographic and clinical characteristics among women with and without obesity are shown in - Table 1. Women with obesity were more likely to be Black, have gestational diabetes, and have chronic hypertension. In addition, while women with obesity continued to have a higher BMI at delivery than women without obesity, women with obesity gained less weight over the course of their pregnancy. Finally, women with obesity were more likely to be induced for maternal indications, while women without obesity were more likely to be induced for fetal indications.

Women with obesity had a higher rate of cesarean delivery than women without obesity (33.3 vs. 23.3\%, $p=0.02$; - Table 2). This difference remained significant when controlling for parity, weight change over pregnancy, and indication for induction (adjusted RR $[\mathrm{aRR}]=1.79,95 \%$ confidence interval [CI]: [1.34-2.39]).

Differences between women with and without obesity in indication for cesarean are also shown in -Table 2. Women with obesity were significantly more likely to undergo a cesarean for failed induction of labor compared with women without obesity ( 47.8 vs. $26.7 \%, p=0.01$ ). This difference also remained significant when controlling for parity, weight change over pregnancy, and indication for induction (aRR $=1.78$ 95\% CI: [1.09-2.92]). Other indications for cesarean, such as arrest of active phase, arrest of descent, and nonreassuring fetal status, were no different between BMI groups.

There was no significant difference in median time to cesarean between women with and without obesity. There was also no significant difference between women with and without obesity in median time to delivery overall or composite maternal morbidity.

\section{Discussion}

This study demonstrates that, even in the context of a standardized labor induction protocol, women with obesity are at higher risk of cesarean delivery. In particular, women with obesity are at increased risk of cesarean for failed induction. The use of a standardized protocol minimizes the effect of care variation related to BMI on labor management. Thus, the continued association between obesity and cesarean, despite standardization, may provide support to a physiologic explanation for the increased risk of cesarean in women with obesity undergoing labor induction.

Many studies have sought to evaluate the physiology of labor in women with obesity by linking obesity with abnormal labor from the perspective of basic science. One study comparing the contractility of myometrium collected during elective cesarean section by maternal BMI demonstrated a significant reduction in the amplitude and frequency of contractions among women with obesity when compared with women with normal weight. ${ }^{17}$ This decrease in contractility is likely secondary to hormonal differences. For example, maternal obesity and high cholesterol are closely related. ${ }^{18}$ Studies have shown that cholesterol and lowdensity lipoprotein (LDL) inhibit spontaneous contractions. ${ }^{19}$ Further, adipokines, such as leptin, visfatin, adiponectin, resistin, and apelin, have also been implicated in the mechanism of decreased uterine contractility. ${ }^{20-24}$

Our findings are consistent with prior work evaluating the relationship between obesity and cesarean delivery. Wolfe et al performed an Ohio birth certificate-based cohort study encompassing 80,887 deliveries comparing rates of failed labor induction by prepregnancy obesity class. In this work, induction failure rates were $13 \%$ in normal-weight women 
Table 1 Demographics and clinical characteristics among women with and without obesity undergoing labor induction using a standardized labor induction protocol

\begin{tabular}{|c|c|c|c|c|}
\hline & & $\begin{array}{l}\text { With obesity }(n=207) \\
n(\%)\end{array}$ & $\begin{array}{l}\text { Without obesity }(n=258) \\
n(\%)\end{array}$ & $p$-Value \\
\hline \multicolumn{5}{|l|}{ Demographics } \\
\hline \multicolumn{2}{|l|}{ BMI at initial prenatal visit $\left(\mathrm{kg} / \mathrm{m}^{2}\right)^{\mathrm{a}}$} & $36.5[32.6-41.1]$ & $24.9[22.1-27.1]$ & $<0.001$ \\
\hline \multicolumn{2}{|l|}{ Maternal age (year) ${ }^{a}$} & $28[23-32]$ & $26[22-32]$ & 0.12 \\
\hline \multirow[t]{4}{*}{ Race } & Black & $180(87.0)$ & $178(69.0)$ & $<0.001$ \\
\hline & White & $20(9.7)$ & $55(21.3)$ & \\
\hline & Asian & $1(0.5)$ & $9(3.5)$ & \\
\hline & Other & $6(2.9)$ & $16(6.3)$ & \\
\hline Ethnicity & Hispanic & $3(1.4)$ & $10(3.9)$ & 0.16 \\
\hline \multirow[t]{2}{*}{ Insurance } & Private & $61(29.5)$ & $99(38.4)$ & 0.05 \\
\hline & $\begin{array}{l}\text { Medicaid/Medicare/ } \\
\text { uninsured }\end{array}$ & $146(70.5)$ & $159(61.6)$ & \\
\hline \multicolumn{2}{|l|}{ Nulliparity } & $112(54.1)$ & $163(63.2)$ & 0.05 \\
\hline \multicolumn{2}{|l|}{ LEEP or CONE } & $7(3.4)$ & $9(3.5)$ & 0.95 \\
\hline \multicolumn{2}{|l|}{ Tobacco use during pregnancy } & $22(10.6)$ & $16(6.2)$ & 0.17 \\
\hline \multicolumn{2}{|l|}{ Gestational diabetes } & $21(10.1)$ & $10(3.9)$ & 0.007 \\
\hline \multicolumn{2}{|l|}{ Pregestational diabetes } & $8(3.9)$ & $3(1.2)$ & 0.07 \\
\hline \multicolumn{2}{|l|}{ Asthma } & $55(26.6)$ & $56(21.7)$ & 0.22 \\
\hline \multicolumn{2}{|l|}{ Chronic hypertension } & $24(11.6)$ & $14(5.4)$ & 0.02 \\
\hline \multicolumn{2}{|l|}{ History of venous thromboembolism } & $5(2.4)$ & $2(0.8)$ & 0.25 \\
\hline \multicolumn{2}{|l|}{ Renal dysfunction } & $2(1.0)$ & $4(1.6)$ & 0.70 \\
\hline \multicolumn{2}{|l|}{ Systemic lupus erythematous } & $1(0.5)$ & $2(0.8)$ & 1.00 \\
\hline \multicolumn{2}{|l|}{ Weight change over pregnancy (lbs) ${ }^{\mathrm{a}}$} & $19[9-27]$ & 27 [17-36.5] & $<0.001$ \\
\hline \multicolumn{2}{|l|}{ Maternal BMI at delivery $\left(\mathrm{mg} / \mathrm{kg}^{2}\right)^{\mathrm{a}}$} & $39.3[36.5-43.1]$ & $29.0[26.9-31.5]$ & $<0.001$ \\
\hline \multicolumn{5}{|l|}{ Clinical characteristics at admission } \\
\hline \multirow[t]{4}{*}{ Indication for induction } & Postdates & $27(13.0)$ & $33(12.8)$ & $<0.001$ \\
\hline & Maternal Indications ${ }^{\mathrm{b}}$ & $82(39.6)$ & $55(21.3)$ & \\
\hline & Fetal Indications ${ }^{c}$ & $75(36.2)$ & $141(54.7)$ & \\
\hline & Elective/other ${ }^{d}$ & $23(11.1)$ & $29(11.2)$ & \\
\hline \multicolumn{2}{|l|}{ Scheduled induction } & $92(44.4)$ & $118(45.7)$ & 0.78 \\
\hline \multicolumn{2}{|l|}{ Gestational age ${ }^{a}$} & $39[38-40]$ & $39[38-40]$ & 0.70 \\
\hline \multirow[t]{3}{*}{ Cervical dilation at induction start } & $<1 \mathrm{~cm}$ & $43(20.8)$ & $46(17.8)$ & 0.51 \\
\hline & $1-2 \mathrm{~cm}$ & $153(73.9)$ & $202(78.3)$ & \\
\hline & $>2 \mathrm{~cm}$ & $11(5.3)$ & $10(3.9)$ & \\
\hline \multicolumn{2}{|l|}{ Modified Bishop's score ${ }^{a}$} & $3[2-4]$ & $3[2-4]$ & 0.11 \\
\hline
\end{tabular}

Abbreviations: BMI, body mass index; LEEP, loop electrical excision procedure.

${ }^{a}$ Median[interquartile range].

bExamples include: chronic hypertension, gestational hypertension, preeclampsia, diabetes, renal disease, history of venous thromboembolism, cardiac disease or other chronic medical condition where induction was recommended.

'Examples include: oligohydramnios, intrauterine growth restriction, and abnormality on fetal testing.

'Examples of "other" include history of an intrauterine fetal demise, vaginal bleeding at term, and cholestasis.

compared with $29 \%$ in class-III obesity. ${ }^{9}$ Hermann et al evaluated 12,297 deliveries from the French National Perinatal Survey, comparing cesarean rates by prepregnancy BMI. Here, both obese primiparous and multiparous women with no previous cesarean delivery had increased adjusted
RRs for intrapartum cesarean delivery $(\mathrm{RR}=1.64 ; 95 \% \mathrm{CI}$ : [1.36-1.98]; and $\mathrm{RR}=1.66$; $95 \% \mathrm{Cl}$ : [1.15-2.39], respectively). ${ }^{25}$ While our study is comprised of a smaller sample than prior works, our data provide important support to those analyses. Furthermore, our data substantially add to the 


\begin{tabular}{|c|c|c|c|c|}
\hline & $\begin{array}{l}\text { With obesity } \\
(n=207) \\
n(\%)\end{array}$ & $\begin{array}{l}\text { Without obesity } \\
(n=258) \\
n(\%)\end{array}$ & $p$-Value & $\mathrm{aRR}^{\mathrm{a}}$ \\
\hline Cesarean delivery & $69(33.3)$ & $60(23.3)$ & 0.02 & 1.79 [1.34-2.39] \\
\hline \multicolumn{5}{|l|}{ Indication for cesarean ${ }^{\mathrm{b}}$} \\
\hline Failed induction & $33(47.8)$ & $16(26.7)$ & 0.01 & 1.78 [1.09-2.92] \\
\hline Arrest of active phase & $15(21.7)$ & $17(28.3)$ & 0.39 & $0.73[0.40-1.33]$ \\
\hline Arrest of descent & $6(8.7)$ & $7(11.7)$ & 0.58 & $0.85[0.30-2.37]$ \\
\hline Nonreassuring fetal status & $33(47.8)$ & $34(56.7)$ & 0.32 & $0.90[0.65-1.26]$ \\
\hline Elective/other ${ }^{c}$ & $8(11.6)$ & $8(13.3)$ & 0.77 & $0.66[0.24-1.82]$ \\
\hline Dilation of amniotomy $(\mathrm{cm})^{d}$ & $4(4.5)$ & $4(4.5)$ & 0.81 & - \\
\hline Time to amniotomy $(\mathrm{h})^{\mathrm{d}}$ & $6.9[4.0-11.3]$ & $6.7[3.6-10.9]$ & 0.80 & - \\
\hline Time to cesarean $(h)^{d}$ & $21.6[17.3-30.0]$ & 19.8 [15.6-26.5] & 0.34 & - \\
\hline Time to delivery $(h)^{d}$ & $16.2[10.6-23.2]$ & $15.4[10.3-20.8]$ & 0.13 & - \\
\hline Composite maternal morbiditye & $17(8.2)$ & $15(5.8)$ & 0.31 & - \\
\hline
\end{tabular}

Abbreviation: aRR, adjusted relative risk.

${ }^{a}$ Adjusted for parity, weight change over pregnancy, and indication for induction.

b If listed as primary or secondary indication.

"Examples of "other" include malpresentation diagnosed during induction and worsening preeclampsia remote from delivery.

${ }^{\mathrm{d}}$ Median [interquartile range].

$e_{\geq 1}$ of the following during labor, delivery, or in the 4 weeks postpartum: third/fourth-degree perineal laceration, blood transfusion, endometritis, wound separation-infection (defined by the need for additional wound closure or the need for antibiotics), venous thromboembolism, hysterectomy, intensive care unit admission, or death.

current body of literature by demonstrating that differences in rates of cesarean delivery and failed induction remain for women with obesity despite standardized labor management.

\section{Strengths and Limitations}

This study has several strengths. Our work was performed as a secondary analysis of randomized trial which allowed for a highly controlled environment in which to evaluate outcomes in labor induction. We were able to assess the impact of multiple factors thought to mediate the relationship between obesity and cesarean, such as indication for induction and weight change over pregnancy. We were also able to specifically examine which indications for cesarean were increased among women with obesity. In addition, this study utilized directly measured weight to calculate BMI, limiting classification bias.

However, while the standardized protocol utilized here minimizes provider variation in management, this study is limited in that such practice differences can likely never be eliminated completely. Thus, results might differ if we were truly able to control every aspect of decision-making throughout a labor induction. In addition, protocol adherence was not assessed as a part of this work, thus we were unable to evaluate if clinicians were more likely to deviate from the protocol in women with obesity due to presumptions about vaginal birth success. Furthermore, this work was performed at one, urban, academic center with a fixed sample size, which may limit generalizability. Due to sample size constraints, we were also unable to evaluate obesity with further granularity, such as by IOM class I $\left(B M I=30-34.9 \mathrm{~kg} / \mathrm{m}^{2}\right)$, II $\left(B M I=35-39.9 \mathrm{~kg} / \mathrm{m}^{2}\right)$, and III $\left(B M I \geq 40 \mathrm{~kg} / \mathrm{m}^{2}\right)$.

\section{Conclusion}

While we are beginning to understand the unique physiology of labor and induction in women with obesity from a basic science perspective, the results of this study support the need for additional research. Translational work to develop interventions that target differences in physiology for women with obesity may be beneficial. In addition, future work should evaluate whether we should alter the definitions of arrest disorders for women with obesity, thereby allowing for additional time in labor. Finally, an evaluation should be performed to assess whether standardization of labor induction impacts care variation that occurs due to demographics other than obesity, such as race, where disparate outcomes are less likely physiologic.

\section{Funding}

This study was funded in part by a career development award in Women's Reproductive Health Research, grant no.: K12-HD001265-16 [LL] and T32 Training Grant in Reproductive Epidemiology, grant no.: T32-HD007440 [RH]. 


\section{Conflict of Interest}

None declared.

\section{References}

1 ACOG practice bulletin no 156: obesity in pregnancy. Obstet Gynecol 2015;126(06):e112-e126

2 Lashen H, Fear K, Sturdee DW. Obesity is associated with increased risk of first trimester and recurrent miscarriage: matched case-control study. Hum Reprod 2004;19(07):1644-1646

3 Anderson NH, McCowan LM, Fyfe EM, et al; SCOPE Consortium. The impact of maternal body mass index on the phenotype of preeclampsia: a prospective cohort study. BJOG 2012;119(05):589-595

4 Salihu HM, Dunlop AL, Hedayatzadeh M, Alio AP, Kirby RS, Alexander GR. Extreme obesity and risk of stillbirth among black and white gravidas. Obstet Gynecol 2007;110(03):552-557

5 Kominiarek MA, Vanveldhuisen P, Hibbard J, et al; Consortium on Safe Labor. The maternal body mass index: a strong association with delivery route. Am J Obstet Gynecol 2010;203(03):264. e1-264.e7

6 Marshall NE, Guild C, Cheng YW, Caughey AB, Halloran DR. Maternal superobesity and perinatal outcomes. Am J Obstet Gynecol 2012;206(05):417.e1-417.e6

7 Ellis JA, Brown CM, Barger B, Carlson NS. Influence of maternal obesity on labor induction: a systematic review and meta-analysis. J Midwifery Womens Health 2019;64(01):55-67

8 Roloff K, Peng S, Sanchez-Ramos L, Valenzuela GJ. Cumulative oxytocin dose during induction of labor according to maternal body mass index. Int J Gynaecol Obstet 2015;131(01):54-58

9 Wolfe KB, Rossi RA, Warshak CR. The effect of maternal obesity on the rate of failed induction of labor. Am J Obstet Gynecol 2011;205 (02):128.e1-128.e7

10 Levine LD, Downes KL, Elovitz MA, Parry S, Sammel MD, Srinivas SK. Mechanical and pharmacologic methods of labor induction: a randomized controlled trial. Obstet Gynecol 2016;128(06): 1357-1364

11 O'Driscoll K, Foley M, MacDonald D. Active management of labor as an alternative to cesarean section for dystocia. Obstet Gynecol 1984;63(04):485-490

12 López-Zeno JA, Peaceman AM, Adashek JA, Socol ML. A controlled trial of a program for the active management of labor. $\mathrm{N}$ Engl $\mathrm{J}$ Med 1992;326(07):450-454
13 Frigoletto FD Jr., Lieberman E, Lang JM, et al. A clinical trial of active management of labor. N Engl J Med 1995;333(12):745-750

14 Peaceman AM, Socol ML. Active management of labor. Am J Obstet Gynecol 1996;175(02):363-368

15 Gerhardstein LP, Allswede MT, Sloan CT, Lorenz RP. Reduction in the rate of cesarean birth with active management of labor and intermediate-dose oxytocin. J Reprod Med 1995;40(01):4-8

16 Spong CY, Berghella V, Wenstrom KD, Mercer BM, Saade GR. Preventing the first cesarean delivery: summary of a joint Eunice Kennedy Shriver National Institute of Child Health and Human Development, Society for Maternal-Fetal Medicine, and American College of Obstetricians and Gynecologists Workshop. Obstet Gynecol 2012;120(05):1181-1193

17 Moynihan AT, Hehir MP, Glavey SV, Smith TJ, Morrison JJ. Inhibitory effect of leptin on human uterine contractility in vitro. Am J Obstet Gynecol 2006;195(02):504-509

18 Jie Zhang, Kendrick A, Quenby S, Wray S. Contractility and calcium signaling of human myometrium are profoundly affected by cholesterol manipulation: implications for labor? Reprod Sci 2007; 14(05):456-466

19 Wozniak SE, Gee LL, Wachtel MS, Frezza EE. Adipose tissue: the new endocrine organ? A review article. Dig Dis Sci 2009;54(09): 1847-1856

20 Falcão-Pires I, Castro-Chaves P, Miranda-Silva D, Lourenço AP, LeiteMoreira AF. Physiological, pathological and potential therapeutic roles of adipokines. Drug Discov Today 2012;17(15,16):880-889

21 Barrichon M, Hadi T, Wendremaire M, et al. Dose-dependent biphasic leptin-induced proliferation is caused by non-specific IL6/NF-KB pathway activation in human myometrial cells. Br J Pharmacol 2015;172(12):2974-2990

22 Mumtaz S, AlSaif S, Wray S, Noble K. Inhibitory effect of visfatin and leptin on human and rat myometrial contractility. Life Sci 2015;125:57-62

23 O'Brien M, Earley P, Morrison JJ, Smith TJ. Ghrelin in the human myometrium. Reprod Biol Endocrinol 2010;8:55

24 Lim R, Barker G, Riley C, Lappas M. Apelin is decreased with human preterm and term labor and regulates prolabor mediators in human primary amnion cells. Reprod Sci 2013;20(08):957-967

25 Hermann M, Le Ray C, Blondel B, Goffinet F, Zeitlin J. The risk of prelabor and intrapartum cesarean delivery among overweight and obese women: possible preventive actions. Am J Obstet Gynecol 2015;212(02):241.e1-241.e9 